\title{
Cardiological Signs and Symptoms in Friedreich's Ataxia
}

\author{
M. COTE. A. DAVIGNON, K. PECKO-DROUIN. A. SOlignaC. G. GEOFFroy. B. LEMIEUX AND A. BARBEAU
}

SUMMARY: The cardiovascular signs and symptoms were recorded in 36 patients with typical Friedreich's Ataxia (Group) Ia, Ib). Seventeen patients were asymptomatic and this did not correlate with the severity of the disease. No pathognomonic clinical constellation was found to reveal the underlying cardiomyopathy.

RÉSUMÉ: Une analyse fiat faite des signes et symptomes cardiovasculaires chez 36 patients avec ataxie de Friedreich "rypique" (groupes Ia, Ib). Seuls 17 patients sont asymptomatiques et il ne semble pas y avoir de corrélation avec la sévérité de la maladie. Nous n'avons pas pâ mettre en évidence ane constallation de signes qui serait pathognomonique de la cardiomyopathie sous-jacente de ces patients.
From the Centre Hospitalier Universitaire de Sherbrooke, the Hôpital Ste-Justine in Montreal, the Hopital Hôtel-Dieu in Montreal and the Clinical Research Institute of Montreal.

Reprint requests for the complete supplement on Friedreich's Ataxia to: Dr. André Barbeau. Clinical Research Institute of Montreal, 110 Pine Avenue West, Montreal, H2W IR7 Quebec, Canada.

\section{INTRODUCTION}

In most patients suffering from Friedreich's ataxia, necropsy studies have revealed myocardial involvement. From Friedreich's original cases (1863) to more recent publications (Boyer et al., 1962; Ivemark and Thoren, 1964; Hewer, 1968), the pathological changes include ventricular hypertrophy, some associated with outflow tract obstruction (Ruschhaupt et al., 1972), diffuse myocardial fibrosis, small cell infiltration, and obstructive lesions, especially of small- and medium-size branches of the coronary arteries varying from 10 percent (Hewer, 1968) to 100 percent (James and Fisch, 1963).

The signs and symptoms of this cardiomyopathy vary greatly. They depend not only on the extent of myocardial damage at a given time in the evolution of the disease but also on the severity of the neurological impairment, of the frequently present dorsal scoliosis, and finally on the involvement of the respiratory "pump".

\section{CASE MATERIAL}

Thirty-six patients with typical Friedreich's ataxia (see definition in previous papers) were evaluated by five observers for symptoms and signs of cardiovascular involvement. There were 24 female and 12 male patients. Their ages varied from seven to 35 (median age: 20). The degree of neurological impairment was considered slight (able to walk without support) in three patients, moderate (able to walk with support) in 14, and severe (confined to a wheelchair or bedridden) in $19 \mathrm{pa}-$ tients.

\section{RESULTS (TABLE I)}

Seventeen percent of the patients were subjectively asymptomatic. This group included all patients with slight neurological impairment, but also six patients with severe neurological impairment. The $\cdot$ degree of dorsal scoliosis was minimal to severe $\left(110^{\circ}\right)$. Several reports indicate the presence of cardiovascular symptoms may vary from 10 percent to more than 50 percent, especially in the large necropsy studies (Boyer et al., 1962; Hewer. 1968; Thoren, 1964; Soulie et al., (1965). Fifteen patients complained of slight to moderate dyspnea at rest, but none had severe symptoms of congestive heart failure. Subjective impression of an irregular heart was noted by 13 patients, but atrial flutter or fibrillation was present only in three patients and had been documented transiently in one other. The incidence of artrial tachy-arrythmias, however, may approach 30 percent in fatal cases of Friedreich's ataxia (Ivemark and Thoren, 1964; Schilero et al., 1952; Soulie et al., 1965; Hartman and Boom, 1960). Only three patients had intermittent precordial chest pain, usually oppressive in nature. The two patients with hemodynamically documented obstructive hypertrophic cardiomyopathy did not complain of chest pain or syncope.

Physical examination revealed a rapid heart rate $(90 / \mathrm{min}$. or more) at rest in 20 patients, including two patients with atrial fibrillation. The blood pressure was normal in all and even low in five patients $(90 \mathrm{~mm}$ $\mathrm{Hg}$ or less systolic).

A systolic ejection murmur was audible in 15 of 36 cases, usually grade II or III, of maximum intensity 
TABLE I

\begin{tabular}{|c|c|c|c|c|c|c|c|c|c|c|c|c|c|}
\hline \multirow{2}{*}{ No. } & \multirow{2}{*}{ Patient } & \multirow{2}{*}{ Sex } & \multirow{2}{*}{ Age } & \multirow{2}{*}{$\begin{array}{l}\text { Neurological } \\
\text { Impairment }\end{array}$} & \multicolumn{4}{|c|}{ Symptoms } & \multicolumn{5}{|c|}{ Signs } \\
\hline & & & & & Asymp. & Dysp. & Palp. & Pain & H.R. & BP & Syst. murmurs & Gallop & Scoliosis \\
\hline Group Ia & & & & & & & & & & & & & \\
\hline 1 & C.S. & $\mathrm{M}$ & 22 & Severe & - & $3 / 4$ & + & - & 84 & $120 / 88$ & $2 / 6$ & - & $30^{\circ}$ \\
\hline 2 & I.S. & M & 21 & Severe & + & - & - & - & 104 & $130 / 75$ & - & - & $13^{\circ}$ \\
\hline 3 & F.S. & $\mathrm{F}$ & 25 & Severe & + & - & - & - & 58 & $110 / 70$ & - & 一 & $110^{\circ}$ \\
\hline 4 & S.S. & $\mathrm{F}$ & 15 & Moderate & - & $2 / 4$ & - & - & 80 & $110 / 70$ & $3 / 6$ & S4 & $50^{\circ}$ \\
\hline 5 & G.G. & $\mathrm{F}$ & 21 & Severe & - & - & + & - & 80 & $120 / 74$ & $4 / 6$ & - & $35^{\circ}$ \\
\hline 6 & M.I. & $\mathrm{F}$ & 34 & Moderate & + & - & - & - & 88 & $90 / 65$ & - & - & $32^{\circ}$ \\
\hline 7 & P.R. & $\mathrm{M}$ & 25 & Moderate & - & $2 / 4$ & + & - & 80 & $110 / 60$ & - & S4 & $25^{\circ}$ \\
\hline 8 & M.I. & $\mathrm{F}$ & 22 & Severe & + & - & - & - & 96 & $110 / 70$ & - & - & $35^{\circ}$ \\
\hline 9 & N.I. & $F$ & 8 & Moderate & + & - & - & - & 88 & $105 / 55$ & - & - & $18^{\circ}$ \\
\hline 10 & H.B. & $\mathrm{M}$ & 25 & Severe & - & $2 / 4$ & - & - & 90 & $140 / 70$ & $2 / 6$ & - & $53^{\circ}$ \\
\hline 11 & R.B. & $\mathrm{M}$ & 27 & Severe & - & - & + & - & 110 (A.F.) & $120 / 80$ & - & S3 & $66^{\circ}$ \\
\hline 12 & G.A. & $\mathrm{F}$ & 25 & Severe & + & 一 & - & - & 84 & $125 / 85$ & - & - & $42^{\circ}$ \\
\hline 13 & M.B. & $\mathrm{F}$ & 35 & Severe & - & - & - & + & 94 & $140 / 90$ & $1 / 6$ & - & $13^{\circ}$ \\
\hline 14 & F.F. & $\mathrm{F}$ & 23 & Severe & + & - & - & - & 84 & $116 / 75$ & 一 & - & $80^{\circ}$ \\
\hline 15 & I.S. & $\mathrm{F}$ & 32 & Severe & - & $2 / 4$ & + & - & 150 (A.F.) & $132 / 88$ & - & S3 & $0^{\circ}$ \\
\hline 16 & D.C. & $\mathrm{F}$ & 30 & Severe & + & - & - & - & - & - & - & - & $80^{\circ}$ \\
\hline 17 & S.T. & M & 12 & Moderate & + & - & - & - & 100 & $130 / 80$ & $2 / 6$ & S3 & $5^{\circ}$ \\
\hline 18 & C.D. & $\mathrm{F}$ & 13 & Moderate & + & 一 & - & - & 90 & $110 / 70$ & $2 / 6$ & - & $40^{\circ}$ \\
\hline 19 & D.D. & $\mathrm{F}$ & 17 & Moderate & + & - & - & - & 80 & $120 / 70$ & - & S3 & $5^{\circ}$ \\
\hline 20 & A.B. & M & 19 & Moderate & + & - & - & - & 64 & $110 / 80$ & $2 / 6$ & - & $11^{\circ}$ \\
\hline 21 & G.L. & $M$ & 17 & Moderate & - & $2 / 4$ & - & - & 98 & $130 / 90$ & $3 / 6$ & $\mathrm{~S} 3$ & $20^{\circ}$ \\
\hline 22 & M.H. & $F$ & 13 & Moderate & + & - & - & 一 & 90 & $105 / 85$ & $3 / 6$ & - & $9^{\circ}$ \\
\hline 23 & G.H. & $M$ & 26 & Severe & - & $3 / 4$ & + & - & 150(A.F.) & $90 / 70$ & - & S4 & $40^{\circ}$ \\
\hline 24 & M.H. & $\mathrm{F}$ & 25 & Severe & - & $2 / 4$ & + & - & 100 & $110 / 80$ & $2 / 6$ & 一 & $120^{\circ}$ \\
\hline 25 & L.D. & $\mathrm{F}$ & 12 & Moderate & + & - & - & - & 85 & $100 / 70$ & - & - & $13^{\circ}$ \\
\hline 26 & J.D. & $\mathbf{F}$ & 9 & Moderate & + & - & - & - & 100 & $110 / 60$ & - & - & $18^{\circ}$ \\
\hline 27 & J.C. & $\mathrm{F}$ & 13 & Slight & + & - & - & - & 105 & $110 / 70$ & - & - & $18^{\circ}$ \\
\hline 28 & S.C. & $F$ & 9 & Slight & + & - & - & - & 90 & $100 / 65$ & - & - & $15^{\circ}$ \\
\hline 29 & Y.M. & $\mathbf{M}$ & 16 & Moderate & - & $2 / 4$ & - & - & 80 & $130 / 80$ & - & $\mathrm{S} 4$ & $32^{\circ}$ \\
\hline 30 & G.M. & M & 12 & Moderate & - & $2 / 4$. & - & - & 85 & $110 / 80$ & $3 / 6$ & - & $30^{\circ}$ \\
\hline 31 & D.T. & M & 17 & Severe & - & $2 / 4$ & + & - & 70 & $90 / 45$ & - & S3 & $68^{\circ}$ \\
\hline 32 & M.T. & $\mathrm{F}$ & 23 & Severe & - & $2 / 4$ & + & - & 100 & $110 / 80$ & - & - & $65^{\circ}$ \\
\hline 33 & F.T. & $\mathrm{F}$ & 28 & Severe & - & $2 / 4$ & + & - & 100 & $130 / 85$ & - & S3 & $60^{\circ}$ \\
\hline \multicolumn{14}{|l|}{ Group Ib } \\
\hline 1 & L.F. & $F$ & 15 & Severe & + & - & - & - & 80 & $100 / 60$ & - & S3 & $50^{\circ}$ \\
\hline 2 & D.F. & $\mathrm{F}$ & 14 & Moderate & - & $2 / 4$ & + & - & 90 & $85 / 60$ & $2 / 6$ & - & $78^{\circ}$ \\
\hline 3 & B.F. & $\mathrm{F}$ & 7 & Slight & + & - & - & - & 76 & $90 / 50$ & $2 / 6$ & $\mathrm{~S} 4$ & $28^{\circ}$ \\
\hline
\end{tabular}

at the left sternal border. In 14, a diastolic third or fourth heart sound was present on auscultation. It has been noted that the heart murmur may disappear with time (Thoren, 1964).

In two patients with documented left ventricular hypertrophic obstructive cardiomyopathy, the carotid pulse recording (Figure 1, Elias et al., 1972) demonstrated the typical rapid initial upstroke and mid-systolic retraction at the maximum intensity of the systolic murmur and prominent " $A$ " wave on the apex cardiogram.

Previous reports recorded similar findings: frequent systolic ejection murmurs (Soulie et al., 1965; Thoren, 1964; Boyer et al., 1962) and diastolic heart sounds in 5 to 25 percent of cases. It is interesting to note that there does not appear to be a clear correlation between the severity of the cardiac symptoms or presence of significant clinical find- ings with the degree of neurological impairment. Indeed, in one reported case (Ruschhaupt et al., 1972), congestive heart failure secondary to an obstructive cardiomyopathy anteceded significant neurological signs or symptoms. However, of the reported patients who died (usually between the ages of 20 and 30), cardiopulmonary complications were frequent, with arrythmias, congestive heart failure, and pulmonary and cerebral emboli pre- 


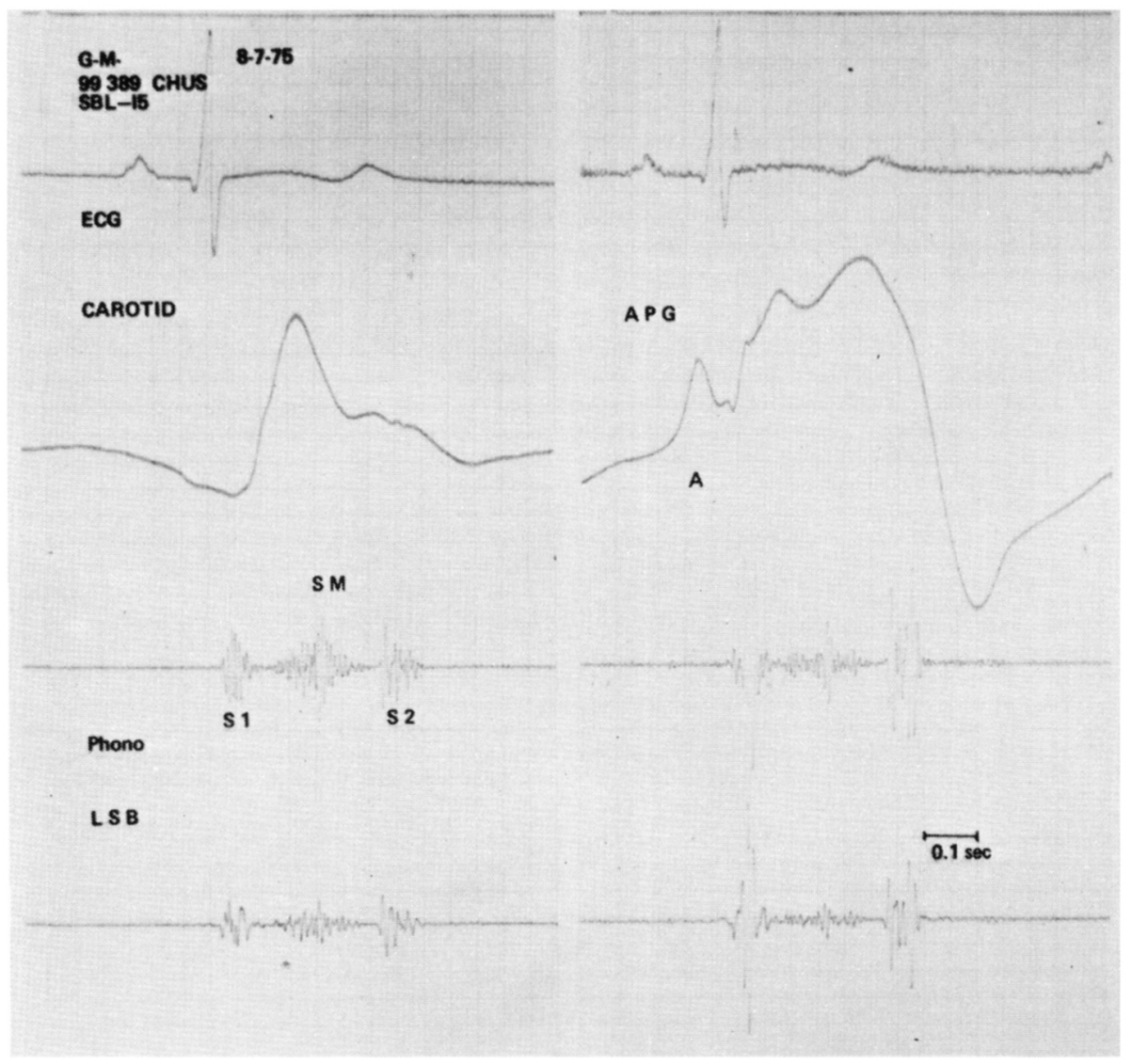

Figure $I-$ ECG=electrocardiogram. Carotid = carotid pulse recording. $\mathrm{S}_{1}, \mathrm{~S}_{2}=$ first and second heart sound. S.M.= systolic murmur. Phono=phonocardiogram. L.S.B. =left sternal border. A.P.G.= apex cardiogram. $A=$ " $A$ " wave.

dominating (Hewer, 1968; Thoren, 1964; Ivemark and Thoren, 1964; Boyer et al., 1962).

\section{CONCLUSION}

An hypertrophic cardiomyopathy, concentric or asymmetric, is frequently present in patients afflicted with Friedreich's ataxia and can often be detected clinically as shown in the present study. It is difficult to assess the degree of myocardial involvement. Phonocardiograms and echocardiograms will help in identifying patients with significant obstructive hypertrophic cardiomyopathy, but cardiac catheterization may be required in order to evaluate more thoroughly symptomatic patients.

\section{ACKNOWLEDGMENTS}

These studies were supported in part by I'Association Canadienne de l'Ataxie de Friedreich, La Fondation Jeanne-Mance and the Medical Research Council of Canada.

\section{REFERENCES}

BOYER, S. H., CHISHOLM, A. W. and McKUSICK, V. A. (1962). Cardiac aspects of Friedreich's ataxia. Circulation, 25. 493-505.

ELIAS, G., GUERIN, R., SPITAELS, S., FOURON, J. C., and DAVIGNON, A. (1972). Sténose musculaire sous-aortique et ataxie de Friedreich. Union Méd. Can.. 101, 474-478.

FRIEDREICH, N. (1863). Ueber degenerative atrophie der spinalen hinterstränge. Arch. Pathol. Anat. Physiol. Klin. Med.. 26, 391-419, 433-459.

HARTMAN, J. M., and BOOTH, R. W. (1960). Friedreich's ataxia: A neuro-cardiac disease. Ame. Heart J., 60, 716-720.

HEWER, R. L. (1968). Study of fatal cases of Friedreich's ataxia. Brit. Med. J., 3, 649-652.

IVEMARK, B., and THOREN, C. (1964). The pathology of the heart in Friedreich's ataxia - Changes in coronary arteries and myocardium. Acta Med. Scand., 175, 227-237.

JAMES, T. N., and FISCH, C. (1963). Observations on the cardio-vascular involvement in Friedreich's ataxia. Amer. Heart J., 66, 164-175.

RUSCHHAUPT, O. G.. THILENIUS, $O$. G., and CASSELS, D. E. (1972). Friedreich's ataxia associated with idiopathic hypertrophic subaortic stenosis. Amer. Heart J., 84, 95-102.

SCHILLERO, A. J., ANTZIG, E., and DUNN, J. (1952). Friedreich's ataxia and its cardiac manifestations. Amer. Heart J., 44, 805-822.

SOULIE, P., VERNANT, P.. COLONNA. D., and GRYNBERG, J. C. (1965). Aspects cardiaques de la maladie de Friedreich. Coeur et médicine interne, 4, 311-319.

THOREN, C. (1964). Cardiomyopathy in Friedreich's ataxia; with studies of cardiovascular and respiratory function. Acta Paediatrica (Suppl.), 53, 153, 1-36. 